Original Research Paper

\title{
Hippocampal Pyramidal Cell Degeneration and Changes in CREB Protein in Datura stramonium Treated Rats
}

\author{
${ }^{1}$ Peter Etim Ekanem, ${ }^{2}$ Dare S. Sunday, ${ }^{3}$ Sunday Abba and ${ }^{4}$ Amanuel Tesfay \\ ${ }^{1}$ Anatomy Unit, Biomedical Department, Mekelle University, Ethiopia \\ ${ }^{2}$ Anatomy, Faculty of Biomedical Sciences, Kampala International University, Uganda \\ 3,4 Anatomy, Biomedical Institute, College of Health Sciences, Mekelle University, Mekelle, Ethiopia
}

\author{
Article history \\ Received: 17-07-2015 \\ Revised: 20-08-2015 \\ Accepted: 11-09-2015 \\ Corresponding Author: \\ Peter Etim Ekanem \\ Anatomy Unit, Biomedical \\ department, Mekelle \\ University, Ethiopia \\ Email: etimakpan@gmail.com
}

\begin{abstract}
Datura Stramonium (DS) is a tropical shrub which is available worldwide. It has various uses and is often used to increase the intoxication of certain beverages. The seeds of this plant are very toxic but are commonly smoked in like manner as tobacco. The present study investigated the potential harmful effects of DS on pyramidal cells and CREB protein in the hippocampus of Wistar rats in order to further elucidate the effects of DS seed extract on hippocampal structure. The study was conducted on both male and female Wistar rats (200-250 g). They were first divided into three batches, which were further sub-divided into four groups in each batch with eight animals per group. Ethanolic extract of dried seeds of DS was diluted in normal saline and given to the treatment groups. The treated groups received intraperitoneal administration (i.p.) of $750 \mathrm{mg} \mathrm{kg}^{-1}$ (Bania et al., 2004) of diluted DS seed extracts, once in batch 1 , twice in batch 2 and thrice in batch 3 per day respectively for 4 weeks while the control groups received an equivalent of normal saline. The rats were euthanized and Western blot analysis used to evaluate the levels of CREB protein in the rats' hippocampi. Sections of each hippocampus were histologically processed in all the groups and silver impregnation stain for degenerating axons and neurons was used to elucidate the actions of DS on the pyramidal neurons of the hippocampus. The result of i.p. administration of DS extract $\left(750 \mathrm{mg} \mathrm{kg}^{-1}\right)$ given three times per day to the treated rats showed significant histological changes such as axonal atrophy, cytoplasmic vacuolation and neuronal necrosis of the pyramidal cells of the hippocampus, as well as an increase in CREB protein levels in both male and female rats. Excessive ingestion of DS seeds, therefore, may lead to hippocampal pyramidal cell losses and an increase in CREB protein levels in the hippocampus. This may be implicated in neurological disorders.
\end{abstract}

Keywords: Datura stramonium, Hippocampus, Neuronal Atrophy, CREB Protein, Pyramidal, Histological Changes

\section{Introduction}

Datura stramonium, also known as Jimson weed, is a common weed found along roadsides, in cornfields, pastures and waste areas. It is a cosmopolitan weed of worldwide distribution. It has been used in some cultures to treat cough, chest pain, asthma, impotence and diarrhea as well as as an analgesic for the control of pain and to kill parasites (Parotta, 2001). It has been smoked in the same way as tobacco and is used in many cultures worldwide in alcoholic beverages to increase intoxication (Wagner et al., 1998). It has also been considered as an alternative medicine in the treatment of Parkinson's disease. The well known alkaloids in this plant include: Hyoscine, atropine, scopolamine and hyoscyamine (Arnett, 1995). The toxins in Jimson weed are Tropane belladonna alkaloids which possess strong anticholinergic properties (Arnett, 1995; Haddad and Winchester, 1990; Brown, 1990). It has been documented to contain gamma -1-glutamyl -1-aspartate which impairs learning (Schmitz-Bourgeois et al., 1988). 
The hippocampus is part of the hippocampal formation along with the dentate gyrus, subiculum etc. In the human brain, the hippocampus and dentate gyrus are deep in the temporal lobe, parallel to, but under the cover of the parahippocampal gyrus, separated from it by the hippocampal sulcus. The functions in which the hippocampal formation is involved are increasing. These include: Memory functions, motivational and attentional mechanisms, inhibitory processes, aggressive behaviour, neuro-endocrine activities etc (Heimer, 1983). The belief that the hippocampus is important for memory is based on careful examination of patients with memory loss as a result of brain damage. The degree and type of memory deficits were correlated with the site of the brain lesion, as determined histologically, after the death of the patient. Such lesions involve the hippocampal formation as the most constant finding (Brodal, 2004).

The hippocampus is basically a three layered strip of archicortex. The ventricular surface of the hippocampus, the alveus, is smooth and white. The alveus contains heavily myelinated axons, principally those of hippocampal pyramidal neurons. From the alveus inward is: The polymorphic layer, the pyramidal cell layer and the molecular layer. The middle layer of the hippocampus, the pyramidal layer, contains three to four rows of densely packed pyramidal cells. These pyramidal cells are frequently called bitufted neurons or double pyramid, because of the large basal dendrites that extend deep into the polymorphic layer. The axons of the pyramidal cells descend through the polymorphic layer to reach the more superficial alveus.

The large pyramidal cells of the pyramidal layer are the most characteristic cell type in the hippocampus. These large neurons have basal dendrites and the axons of these neurons descend into the polymorphic layer (Burt, 1993).

Special properties of the pyramidal cells make these neurons susceptible to various toxins both in the brain and in the blood. Some of these properties include the perpendicular pattern of the dendrites to the cortical surface, resulting in different layers of cortex impinging at different points along the dendritic tree and the pyramidal dendrites containing dendritic spines that amplify currents (inputs) so that distant synaptic sites can more easily generate action potentials (Burt,1993). Therefore, to study the effects of DS on the morphology of hippocampal pyramidal cells would provide insight into its activity on brain cells.

One of the cellular transcription factors found in the neurons is CREB (cAMP response element-binding protein). CREB is also important for the survival of neurons, as shown in genetically engineered mice, where CREB and CREM were deleted in the brain. If CREB is lost in whole developing mouse embryos, the mice die immediately after birth, again highlighting the critical role of CREB in promoting survival (Bear et al., 2015). CREB also has an important role in the development of drug addiction (Wang et al., 2009. Therefore, drugs can affect CREB levels, which in turn can affect the survival or degeneration of axons.

The range of toxicity of the DS seed is highly variable and unpredictable because the concentration of specific alkaloids varies with species, cultivation, environment, temperature, moisture and storage (Goldfrank, 1994). This contributes to the danger of its misuse since the dosage cannot be predicted (Haddad and Winchester, 1990). This work is aimed at investigating the harmful potential effect of DS seed extract on the pyramidal cells and CREB protein levels in the hippocampus. This will further demonstrate the effect of DS seeds on the structure and morphology of the hippocampal pyramidal cells.

\section{Materials and Methods}

\section{Plant Material Handling}

Datura stramonium seeds were harvested in Samaru, Zaria, Nigeria and identified in the herbarium of Biological Sciences, Ahmadu Bello University and the Department of Pharmacognosy, Faculty of Pharmaceutical Sciences, A.B.U., Zaria. The seeds were air-dried, extracted with $96.5 \%$ ethanol and phytochemical analysis conducted to identify the chemical composition of the extract. Dragendorff's reagent was used to confirm the presence of the major alkaloids and Thin Layer Chromatography (TLC) was used to identify scopolamine, hyoscine, atropine and hyoscyamine as the major alkaloids.

\section{Animal Treatments}

Adult male and female Wistar rats, weighing 200g$250 \mathrm{~g}$ were used in this study. The animals were obtained from the animal houses of the Anatomy department and the Veterinary Faculty, both of A.B.U., Zaria. The rules and regulations governing animal handling of Ahmadu Bello University were strictly adhered to and the experiment was conducted in accordance to the ethical committee guidelines. A total of 96 experimental animals were randomly assigned to three batches, which were further sub-divided into four groups in each batch with eight animals per group. Groups $\mathrm{A}$ and $\mathrm{C}$ served as controls for both male and female rats in each batch and received normal saline of $750 \mathrm{mg} \mathrm{kg}{ }^{-1}$ only. Bania et al. (2004) proposed a pretreatment dose of $7.5 \mathrm{mg} \mathrm{kg}^{-1}$ atropine equivalent of Datura stramonium seed extract as a protective agent in severe organophosphate toxicity. This was used as a reference for the calculation of the treatment doses for each batch of treated rats. To induce intoxication, 7.5 $\mathrm{mg} \mathrm{kg}^{-1}$ was increased tenfold since $\mathrm{LD}_{50}$ was found to be above $5000 \mathrm{mg} \mathrm{kg}^{-1}$. Groups B and D were treated with different doses of DS extract. Batch 1 received 
$750 \mathrm{mg} \mathrm{kg}{ }^{-1}$, i.p. once daily, batch 2 received $750 \mathrm{mg}$ $\mathrm{kg}^{-1}$, i.p. twice daily and batch 3 received $7.5 \mathrm{mg} \mathrm{kg}^{-1}$, i.p. thrice daily. This treatment was continued for 4 weeks, after which the animals were sacrificed by decapitation. Their brains were dissected on ice and the hippocampus removed and stored at $-80^{\circ} \mathrm{C}$ while some pieces of the brain were fixed in $10 \%$ formalin for histological processing.

\section{Histological Tissue Processing for Microscopy}

This part of the work was carried out in the histology unit of the Anatomy department at A.B.U., Zaria, Nigeria. The brain tissues were dehydrated in different grades of alcohol and cleared in xylene using an automatic processing machine (Shandon Southern Duplex Processor). The tissues were then infiltrated with paraffin wax and blocked in the coronal plane. Serial sections of the blocks were taken at $8 \mu \mathrm{m}$ with a (Leitz Wetzlar) microtome, mounted on glass slides and allowed to dry overnight. The staining technique employed was Glee's ammonia silver impregnation method for neurons and degenerating axons in paraffin sections (Lillie, 1965). The pyramidal cells and neurites stained black. Tissues were observed in upright life science microscope (Olympus $\times 1000)$ with an attached digital camera. Sections of the hippocampus were observed at low and high magnification for changes in pyramidal neurons of the hippocampus.

\section{Western Blot Analysis of CREB Protein in the Hippocampus}

This part of the work was carried out in the molecular biology lab at Makerere University, Uganda. $0.958 \mathrm{~g}$ of hippocampal brain tissues used for Western blotting in both males and females were homogenized using a sonicator machine (MSE) in the working buffer (1mM PIPES, $0.1 \mathrm{mM}$ EDTA, $1 \mathrm{mM}$ EGTA, 1mM PMSF, Tris-choride, acrylamide and $0.1 \%$ protease inhibitor). The homogenates were poured back into a centrifuge tube and spinned with a micro-centrifuge (Sorvail RF Micro centrifuge) at $100,000 \mathrm{rpm}$ at $4^{\circ} \mathrm{C}$ for $10 \mathrm{~min}$. The clear supernatant was extracted with a micropipette and dispensed into smaller glasses. Two gels were prepared: One gel for staining with Coomasie blue and the other for Western blot. The two gels were loaded at the same time to give the same experimental conditions. Protein concentration was determined using Bicinchonic Acid Assay (BCA) kit (Pierce Biotechnology). A total of 40 $\mu \mathrm{g}$ of total protein from each sample was applied for western blot. Protein samples were denatured and dissolved in $8 \%$ SDS-PAGE then transferred to Nitrocellulose membrane. After the transfer, the membrane was removed from the sandwich and rinsed in distilled water. The membrane was stained for 30 sec with ponceaus stain to verify the effectiveness of the transfer. It was immediately washed three times in $0.1 \% 20$ in Phosphate Buffer Saline (PBS).

The membrane was then placed in a glass plate and blocked at room temperature in blocking solution (Bovine serum albumin, 20, PBS and 5\% nonfat milk) and incubated overnight at $4{ }^{\circ} \mathrm{C}$. The blocking solution was poured away and the membrane washed three times with PBS. The primary antibody CREB -1(C21) rabbit polyclonal antibody (SC-186 -Santa Cruz Bio technology) was added at a dilution of 1:200 according to the manufacturer's recommendation and allowed to incubate for $1 \mathrm{~h}$ at room temperature with gentle agitation.

Removal of unbound primary antibody was done by washing the membrane in PBS three times at room temperature. The secondary antibody i.e., Goat-rabbit $1 \mathrm{gG}$ coupled to horse radish peroxidase (sigma) was diluted 1:10000 in antibody solution. The membrane was then incubated in a rocking platform for one hour at room temperature.

The membrane was developed in hydrogen peroxide (Sigma co.) and the blot on the membrane captured in Enhanced Chemiluminescence (New Life Science Products, Boston, MA). Films were exposed for $10 \mathrm{sec}$ and developed. Protein levels were normalized before immunoprecipitation by DC assay (Bio-Rad) and prominent background bands.

\section{Results}

Effects of Datura stramonium on the Histology of the Pyramidal Cells of the Hippocampus

Batches 1 and 2 of the treated rats did not show any significant changes in the pyramidal cells of hippocampus when they were compared with the control groups.

Batch 3 treated groups showed significant changes in the pyramidal cells of the hippocampus for both male and female rats.

There was no comparison made in the differences of neuronal losses between the male and female apart from those observed under the microscope.

The changes observed in the treatment groups as compared to the control groups included: Axonal atrophy, cytoplasmic vacuolation, necrosis and cell loss.

\section{Effect of Datura stramonium Extract on the CREB Protein of the Hippocampus}

A brain fraction prepared from the hippocampus of each experimental group was subjected to immunoblotting with an antibody specific for CREB protein. There was a significant increase in the protein expression of CREB in all the treated groups. 


\section{Discussion}

This present study investigated the potential effects of DS extract on the pyramidal cells of the hippocampus. The micrograph of the batch 3 treated group in male rats (Plate 3 ) indicated that DS extract was found to cause atrophy of the axons and fibres, vacuolation, cell necrosis and cell losses as compared to the controls in Plates 1 and 2. In the females, Plate 6 showed similar changes as seen in the male treated rats (batch 3), compared to the controls in Plates 4 and 5. Stella et al. (1997), showed in their work that mice lacking the serine protease tissue Plasminogen Activator (tPA) were resistant to excitotoxin-mediated hippocampal neuronal degeneration, implicating an extracellular proteolytic cascade in the degeneration of hippocampal neurons. This is consistent with the study carried out by Bihaqi et al. (2012) in which the histology of the cerebral cortex examined under light microscope showed increased neuronal loss, ghost cells, hemorrhage and vacuolated cytoplasm on treated rats that received daily intraperitoneal administration of scopolamine. Scopolamine is one of the main alkaloids identified in the DS seed extract as an active component and may have caused the histological changes observed in the pyramidal cells of the hippocampus. Further evidence can be supported by the fact that various mediators can contribute to excitotoxin action, including production of reactive oxygen intermediates, nitric oxide, p53 and cytokines which could lead to a series of cascading events leading to cell losses in the hippocampus (Coyle and Puttfarcken, 1993; Lipton and Rosenberg, 1994; Ankarcrona et al., 1995; Morrison et al., 1996). The implications of several of these toxic effects on neuronal degeneration in the hippocampus have been widely investigated. DS tropane alkaloids all have a long duration of effect, cross the blood brain barrier and have central anti-cholinergic effects (Bania et al., 2004) which induce strong hypnosis and can also induce neuronal degeneration (Hughes and Clark, 1939).

The SDS-PAGE in Fig. 1 was used to detect the CREB protein at $43 \mathrm{kDa}$. CREB Protein level was elevated in this study in all the treated male and female groups when compared with the controls as seen in Fig. 2. The observed result does not tally with the theory of depressed CREB expression in the hippocampus associated with neurodegeneration. Dragana et al. (2009) in their analysis of the hippocampus of A-CREB mice revealed a massive loss of neurons in the CA1 subfield, most likely as a consequence of the dramatic and sustained inhibition of CREB function in pyramidal neurons. They concluded that long term chronic inhibition of CREB function caused severe loss of neurons in the CA1 subfield of the hippocampus. CREB has been shown to be important for the survival of neurons in genetically engineered mice. Evidence has shown that its under-functioning is associated with Major Depressive Disorder (MDD). In addition, postmortem examinations of cortices of patients with untreated MDD contain reduced concentration of CREB compared to both healthy and patients treated with anti-depressants. This reduction in CREB was also associated with neuronal losses (Blendy, 2006). There are two forms of CREB: CREB-2 represses gene expression when it binds to the CRE; CREB- 1 activates transcription, but only when it is phosphorylated by Protein Kinase A (PKA) (Bear et al., 2015).

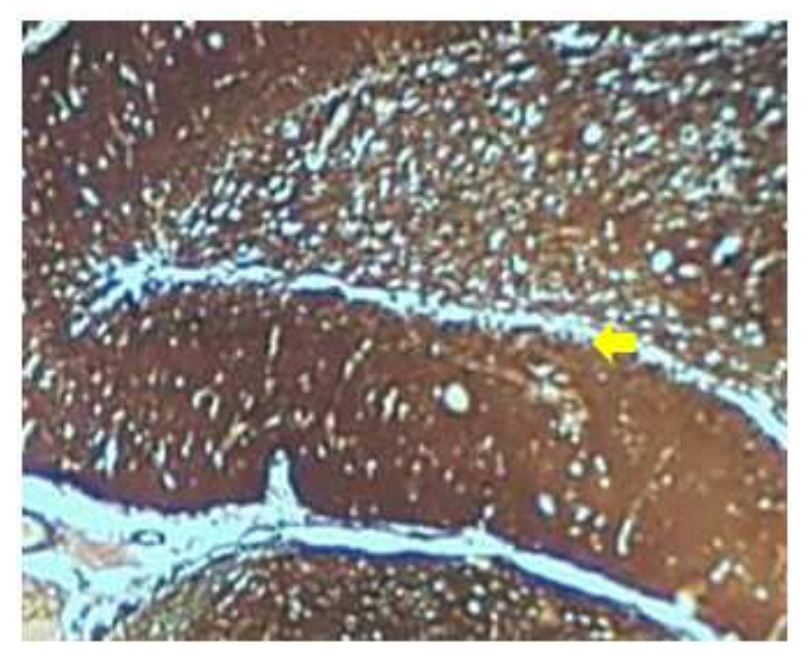

Plate 1. Coronal section of control male rat showing layers of the hippocampus and the pyramidal cell layer (yellow arrow) Silver stain. $\times 400$

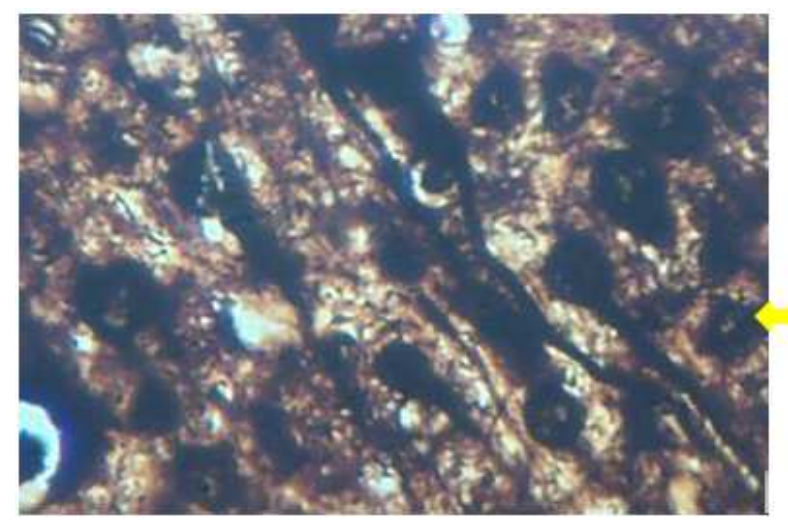

Plate 2. Shows magnified section of the prominent pyramidal neurons (yellow arrow), axons and dendrites in the hippocampus of control male rat Silver stain. $\times 400$ 


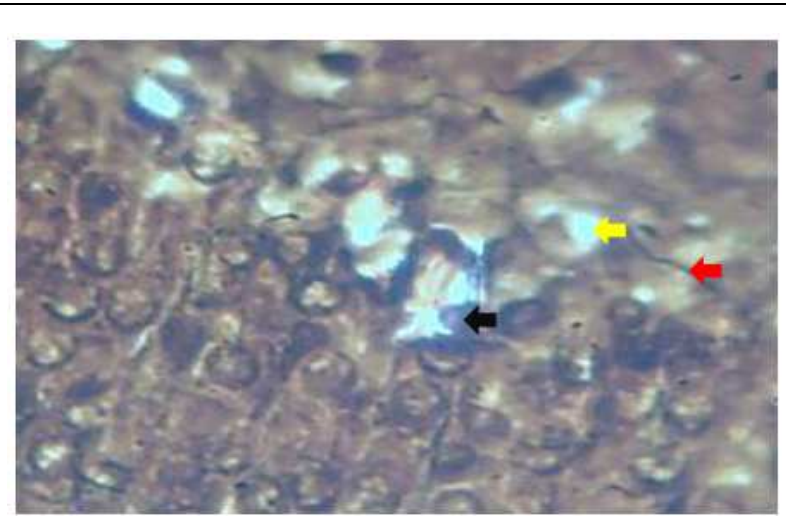

Plate 3. Batch 3 male treated rat hippocampus showing: necrotic cells (black arrow), axonal atrophy (red arrow) and cytoplasmic vacuolation (yellow arrow) of the pyramidal cells. Silver stain $\times 400$

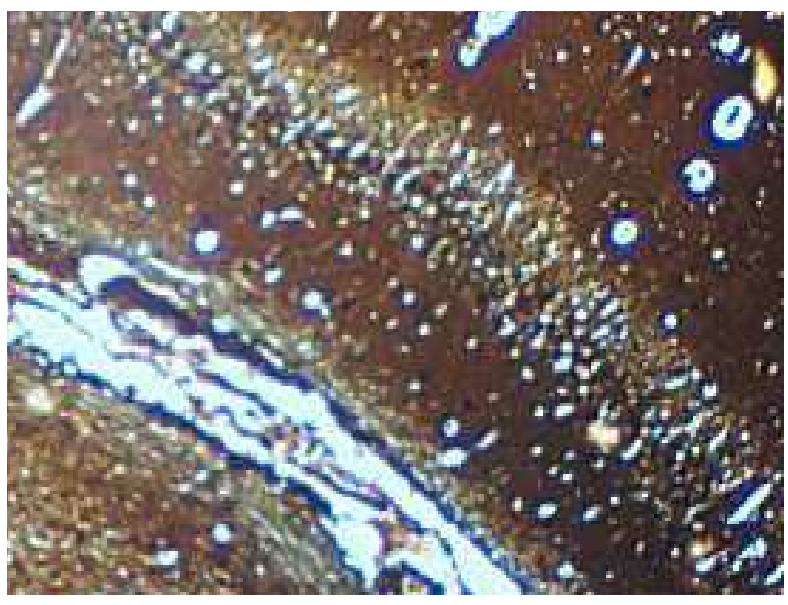

Plate 4. Coronal section of control female rat showing pyramidal layer (yellow arrow) of the hippocampus. Silver stain. $\times 400$

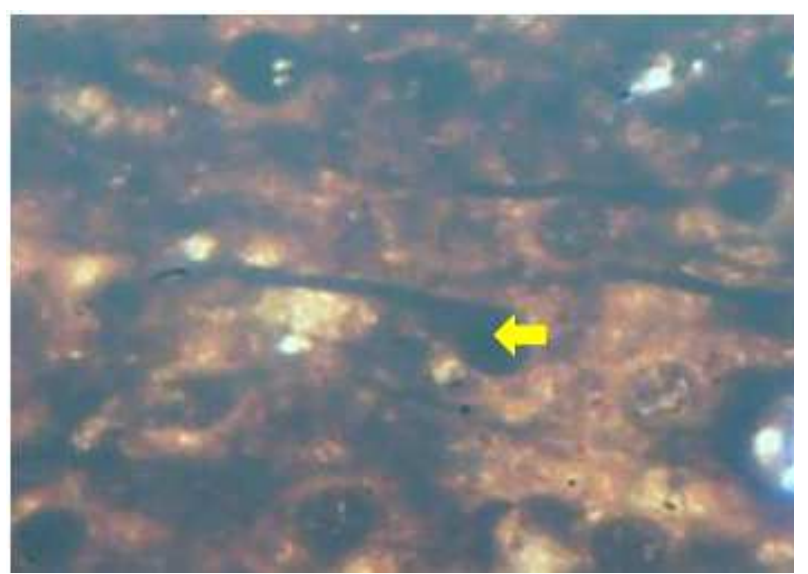

Plate 5. Magnified section of the prominent pyramidal neurons (yellow arrow), axons and dendrites in the hippocampus of control female rat Silver stain. $\times 400$

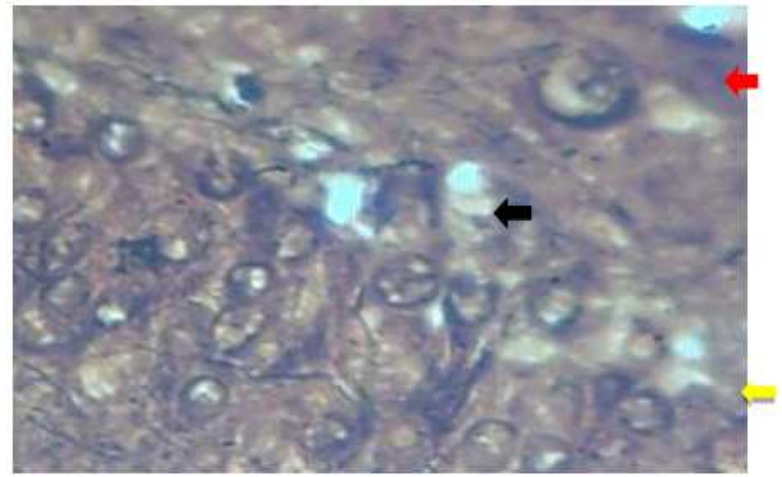

Plate 6. Batch 3 female treated rat's hippocampus showing: Necrotic cells (black arrow) axonal atrophy (yellow arrow and cytoplasmic vacuolation (red arrow) of the pyramidal cells Silver stain. $\times 400$

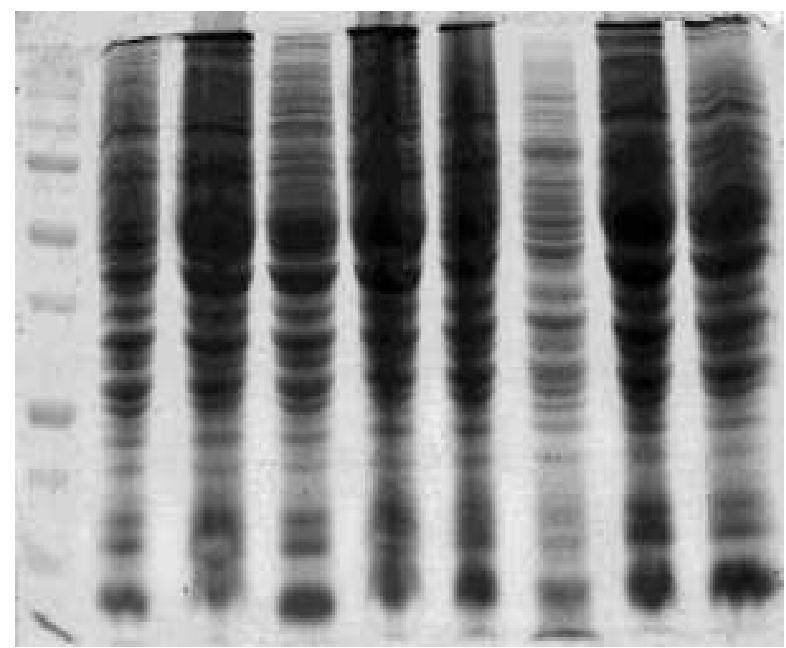

Fig. 1. SDS-PAGE showing protein bands in the hippocampus

$$
\begin{array}{lllllllll}
C M & 1 M & 2 M & 3 M & C F & 1 F & 2 F & 3 F
\end{array}
$$

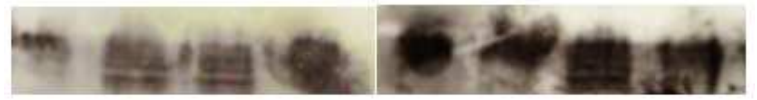

Fig. 2. Western blot of CREB protein in the hippocampus

Flies genetically engineered to overexpress the inactive form of CREB lose their ability to retain long-term memory (Bear et al., 2015).

Differences in expression of CREB in response to exitotoxic drugs have also been observed by several authors just as the one observed in this experiment. Balschun et al. (2003) indicated in their work involving the adult mouse brain that loss of CREB neither prevents learning nor substantially affects performance in some hippocampus-dependent tasks. Furthermore, it spares LTP and LTD in paradigms that are sensitive enough to detect deficits in other mutants. This implies either a species-specific or regionally restricted role of 
CREB in the brain and/or a compensatory upregulation of the cAMP Response Element Modulator (CREM) and other, as yet unidentified, transcription factors (Balschun et al., 2003).

Studies conducted by Gao et al. (2014) on the effects of Cholecystokinin Octapeptide (CCK-8) and its receptor antagonists on cAMP Response Element Binding protein (CREB) and phosphorylated CREB (pCREB) expression in the frontal cortex and hippocampus of rats with morphine withdrawal showed that CREB was expressed in both the cytoplasm and the nucleus. In the pyramidal cell layer of hippocampal CA1 region, CREB showed high expression in the cytoplasm and low expression in the nucleus. This showed that CREB expresses differently in the neurons of hippocampus. In previous studies addressing the role of the transcription factor, cAMP Response Element-Binding protein (CREB) in mammalian long-term synaptic plasticity and memory by gene targeting, the authors asserted that these were compromised by incomplete deletion of the CREB isoforms (Balschun et al., 2003). This could also be true for this experiment, implying that, one of the isoforms of CREB could have been elevated by one of the components of DS.

The atrophy of the axons and neuronal loss may have been caused by inactivation of CREB-1 through dephosphorylation of PKA or increased repressive CREB-2 gene by DS seed extract components leading to increased CREB level in the hippocampus, which could not maintain neuronal structure.

\section{Conclusion}

Excessive administration of DS seed extract may lead to neurite atrophy, vacuolation, necrosis and cell losses of the pyramidal cells in the hippocampus. The histological changes did not correlate with decreases in CREB protein levels, but rather increased levels that could have been triggered by the activation of depressed CREB-2 or dephosphorylation of CREB-1 leading to the non-transcription of CREB-1. This could have contributed to the histological changes observed in the pyramidal cells of the hippocampus with an implication of loss of memory and other neurological disorders that burden several families and the society as a whole.

\section{Acknowledgment}

The researchers wish to acknowledge the staff of the Histology Unit of the Anatomy department of Ahmadu Bello University, Zaria and the Molecular Biology laboratory of Makerere University, Uganda, for their support and providing technical assistance in carrying out this research work.

\section{Funding Information}

The histological part of this work was sponsored by the corresponding author while the molecular aspect was funded by Kampala International University.

\section{Author's Contributions}

Peter Etim Ekanem: Designed the research and its concept, carried out the research, drafted the paper and reviewed it as well as approved the final version for publication.

Dare S. Sunday: Analyzed the data and reviewed the drafted paper for significant intellectual content and approved it for submission.

Sunday Abba: Assisted in writing and reviewing the paper and approved it for submission for publication.

Amanuel Tesfay: Assisted in writing the paper.

\section{Ethics}

The authors strictly observed the ethical guidelines provided in the use of the animals for this research work. No other ethical issues are anticipated.

\section{References}

Ankarcrona, M., J.M. Dypbukt, E. Bonfoco, B. Zhivotovsky and S. Orrenius et al., 1995. Glutamate-induced neuronal death: A succession of necrosis or apoptosis depending on mitochondrial function. Neuron, 15: 961-973. DOI: 10.1016/0896-6273(95)90186-8

Arnett, A.M., 1995. Jimson weed (Datura stramonium) poisoning. Clinical Toxicol. Rev., 18: 1-6.

Balschun, D., D.P. Wolfer, P. Gass, T. Mantamadiotis and H. Welzl et al., 2003. Does cAMP response element-binding protein have a pivotal role in hippocampal synaptic plasticity and hippocampusdependent memory? J. Neurosci., 23: 6304-6314. PMID: 12867515

Bania, T.C., J. Chu, D. Bailes and M. O'Neill, 2004. Jimson weed extract as protective agent in severe organophosphate toxicity. Acad. Emergency Med., 11: 335-338. DOI: 10.1197/j.aem.2003.12.002

Bear, M.F., B.W. Connors and M.A. Paradiso, 2015. Neuroscience: Exploring the Brain. 4th Edn., Lippincott Williams and Wilkins, Philadelphia, ISBN-10: 0781778174, pp: 1008.

Bihaqi, S.W., P.S. Avninder and T. Manisha, 2012. Supplementation pluricaulis attenuates of convolvulus increased tau and Amyloid Precursor Protein (A $\beta$ PP) expression in rat brain. Ind. J. Pharmacol., 44: 593-598. DOI: 10.4103/0253-7613.100383 
Blendy, J.A., 2006. The role of CREB in depression and antidepressant treatment. Biological Psychiatry, 59: 1144-1150. DOI: 10.1016/j.biopsych.2005.11.003

Brodal, P., 2004. The Central Nervous System: Structure and Function. 1st Edn., Oxford University Press, USA, New York, ISBN-10: 0195165608, pp: 515.

Burt, A.M., 1993. Motor Cortex and Descending Motor Pathways. In: Textbook of Neuroanatomy, Burt, A.M. (Ed.), Saunders, Philadelphia, ISBN-10: 0721648991, pp: 322-365.

Coyle, J.T. and P. Puttfarcken, 1993. Oxidative stress, glutamate and neurodegenerative disorders. Science, 262: 689-695. DOI: 10.1126/science.7901908

Dragana, J., L.A. Mikel, M.V. Luis, O. Roman and B. Angel, 2009. Inhibition of cAMP response elementbinding protein reduces neuronalexcitability and plasticity and triggers neurodegeneration. Cerebral Cortex, 19: 2535-2547. DOI: 10.1093/cercor/bhp004

Gao, P., X. Ma, D. Wen, S. Yang and F. Yu et al., 2014. Effects of CCK-8 and its receptor antagonists on expression of CREB and pCREB in prefrontal cortex and hippocampus of morphine withdrawal rats. Chinese J. Pathophysiol., 30: 1158-1165. DOI: $10.3969 /$ j.issn.1000-4718.2014.07.002

Brown, J.H., 1990. Atropine, Scopolamine and Related Antimuscarinic Drugs. In: Goodman and Gilman's the Pharmacological Basis of Therapeutics, Gilman, A.G., T.W. Rall, A.S. Nies and T. Palmer (Eds.), Pergamon Press, New York, ISBN-10: 0080402968, pp: 150-165.

Goldfrank, L.R., 1994. Datura Plant. In: Goldfrank's Toxicologic Emergencies. Goldfrank, L.R. (Ed.), Appleton and Lange, Norwalk, ISBN-10: 0838531466, pp: 103-109.

Haddad, L.M. and J.F. Winchester, 1990. Clinical Management of Poisoning and Drug Overdose. 2nd Edn., Saunders, Philadelphia, ISBN-10: 0721623425, pp: 1557.

Heimer, L., 1983. The Human Brain and Spinal Cord: Functional Neuroanatomy and Dissection Guide. 1st Edn., Springer-Verlag, New York, ISBN-10: 3540907408, pp: 402.
Hughes, J.D. and J.A. Clark, 1939. Stramonium poisoning: A report of two cases. J. Am. Med. Associat., 112: 2500-2502. DOI: 10.1001/jama.1939.02800240016005

Lillie, R.D., 1965. Histologic Technic and Practical Histochemistry. McGraw Hill Book Company, New York, ISBN-10: 9780013652144, pp: 617.

Lipton, S.A. and P.A. Rosenberg, 1994. Excitatory amino acids as a final common pathway for neurological disorders. New England J. Med., 330: 613-622. DOI: 10.1056/NEJM199403033300907

Morrison, R.S., H.J. Wenzel, Y. Kinoshita, C.A. Robbins and L.A. Donehower et al., 1996. Loss of the p53 tumor suppressor gene protects neurons from kainateinduced cell death. J. Neurosci., 16: 1337-1345. PMID: 8778285

Parotta, J.A., 2001. Healing plants of Peninsular India. 1st Edn., CABI Publishing, New York, ISBN-10: 0851995012, pp: 917.

Schmitz-Bourgeois, M., R.I. Amiri, J. Boulanger and Y. Ungerer, 1988. Isolation and structure of a pseudopeptide $\gamma$-l-glutamyl-1-aspartic acid from Datura stramonium that impairs learning retention in mice. Biochimie, 70: 1179-1184. DOI: $10.1016 / 0300-9084(88) 90183-6$

Stella, E.T., D.R. Andrew, H.B. Thomas, L.D. Jay and S. Sidney, 1997. An extracellular proteolytic cascade promotes neuronal degeneration in the mouse hippocampus. J. Neurosci., 17: 543-552. PMID: 8987777

Wagner, A.D., L.S. Daniel, M. Rotte, W. Koutstaal and A. Maril et al., 1998. Building memories: Remembering and forgetting of verbal experiences as predicted by brain activity. Science, 281: 1188-1191. DOI: $10.1126 /$ science.281.5380.1188

Wang, Y., A. Ghezzi, J.C. Yin and N.S. Atkinson, 2009. CREB regulation of $\mathrm{BK}$ channel gene expression underlies rapid tolerance. Genes, Brain Behavior, 8: 369-76. DOI: 10.1111/j.1601-183X.2009.00479.x 